\title{
A Two Element Plasma Antenna Array
}

\author{
F. Sadeghikia \\ Aerospace Research Institute \\ Tehran, Iran \\ sadeghi_kia@ari.ac.ir
}

\author{
F. Hodjat-Kashani \\ Department of Electrical Engineering \\ Iran University of Science and Technology \\ kashani@iust.ac.ir
}

\begin{abstract}
This theoretical study presents the characteristics of plasma monopole antennas in the $\mathrm{VHF} / \mathrm{UHF}$ range using finite difference time domain (FDTD) simulation. Results show that more broadband characteristics can be obtained by increasing the diameter of the plasma tube and that the minor lobes diminish in intensity as diameter increases. Furthermore, the nulls are replaced by low level radiation. Since the collision frequency, which is a function of gas pressure, represents the loss mechanism of plasma, decreasing its value increases the gain and radar cross section (RCS) of the antenna. Theoretical modeling shows that at higher plasma frequencies with respect to the signal frequency, the gain and radar cross section of the plasma antenna are high enough and that the impedance curves are altered as the plasma frequency varies. Using these preliminary studies, mutual impedance and gain of a broadside array of two parallel side-byside plasma elements is presented.
\end{abstract}

Keywords-plasma antenna; radiation pattern; gain; input impedance; surface wave

\section{INTRODUCTION}

Plasma antennas represent a completely new class of antennas which employ ionized gas enclosed in a tube as the conducting element of the antenna. Operating plasma antennas can transmit and receive through a surface wave driven (SWD) plasma column [1-3]. Therefore it could be possible for large stacked antennas with improved range precision to replace numerous small antennas, thus decreasing clutter, weight and interface. Since plasma antennas have properties quite different from those of metallic conductors, the key plasma parameters should be determined and optimized with respect to the characteristics of antennas. However, there are limited numerical simulations in this regard [4-8]. Numerical simulation is a powerful tool to evaluate, with maximum flexibility and minimal cost, the performance of these novel antennas. Numerical analysis of a broadside array of two parallel side-by-side, equal in phase, current plasma elements is the subject of this theoretical study. In the first part of this paper, the radiation characteristics of a SWD plasma monopole antenna are examined, considering the effects of variations of plasma frequency, collision frequency and radius of the tube on the gain, efficiency, input impedance and radar cross section (RCS) of the antenna, because few studies are reported in this regard [4-5]. Further, the primary goals are to investigate efficiently the effects of gas pressure and the excitation power on the performance of a SWD plasma antenna and to provide a better understanding of a linear array of plasma elements. These results should provide more insight to construct an effective array of plasma antennas.

\section{SimUlATION SETUP}

The geometry of the simulated plasma column antenna is illustrated in Figure 1. The plasma column is inserted in a metallic box as a ground plane and a copper ring placed around the tube as a coupling sleeve for launching the signal. The length and radius of the column were chosen to be $600 \mathrm{~mm}$ and $12 \mathrm{~mm}$ respectively and the thickness and height of the coupling sleeve were $3 \mathrm{~mm}$ and $30 \mathrm{~mm}$. The surface wave is launched applying an intense field between the coupling sleeve and the metallic box, and the results are reported in [8]. The dimensions of the metallic box were chosen to be $130 \times 250 \times 55 \mathrm{~mm}$. The gap between the coupling sleeve and the metallic box is $5 \mathrm{~mm}$. The FDTD problem space is $60 \times 80 \times 200$ cells and the waveform of the source is a modulated Gaussian pulse.

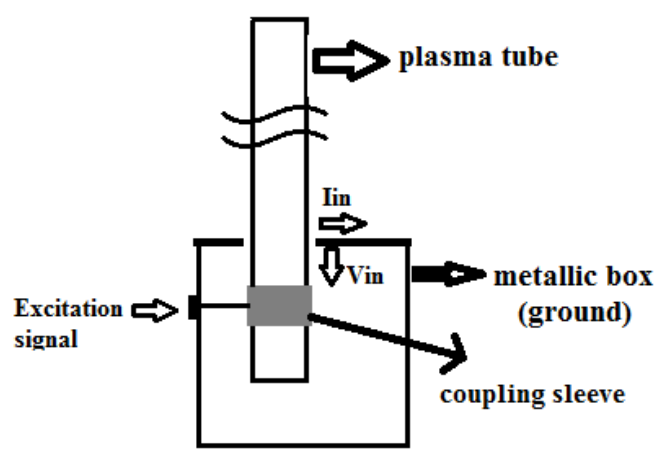

Fig. 1. Simulated plasma column antenna.

The power flux of the wave in a SWD plasma column decreases axially as power is gradually transferred to the discharge $[1,8-10]$ and the electron density steadily decreases from the launcher which has been shown previously experimentally [1] and theoretically [9]. Consequently, the plasma column is axially non uniform and it will result in a non uniform conductivity along the column. This non uniformity of the SWD plasma column is considered as a shortcoming and some remedies have been proposed to overcome it [11]. In this 
investigation, we have considered that the plasma density in the discharge tube remains uniform, which is an ideal condition.

There are many different ways to simulate the electromagnetic interaction in plasma. In this case, the direct integration method (DI) was used to simulate the isotropic cold plasma with a fixed uniform electron density [12]. In this method, the temporal evolution of plasma state is not taken into account and a weak electromagnetic wave propagates in a previously created plasma [6]. In DI method, Maxwell's equations are coupled to an auxiliary ordinary differential equation modeling the response of the current density, $J$, to the field, $E$ as shown in (1)-(3).

$$
\begin{aligned}
& \nabla \times \vec{E}=-\mu_{0} \frac{\partial \vec{H}}{\partial t} \\
& \nabla \times \vec{H}=\varepsilon_{0} \frac{\partial \vec{E}}{\partial t}+\vec{J} \\
& \frac{\partial \vec{J}}{\partial t}+v \vec{J}=\varepsilon_{0} \omega_{p}^{2} \vec{E}
\end{aligned}
$$

Where $\vec{E}$ and $\vec{H}$ are the electric (V/m) and magnetic $(\mathrm{A} / \mathrm{m})$ fields, respectively, $\mu_{0}$ is the magnetic permeability $(\mathrm{H} / \mathrm{m})$ of free space, $\varepsilon_{0}$ is the free space permittivity $(\mathrm{F} / \mathrm{m}), v$ is the average collision frequency $(\mathrm{Hz})$ and $\vec{J}$ is the plasma current density $\left(\mathrm{A} / \mathrm{m}^{2}\right)$. For details of the FDTD methodology, the reader is referred to [8, 13-14].

To calculate the RCS of a plasma antenna, we assume an arbitrary incidence and polarization plane wave as:

$$
E_{i}(r)=(\cos \alpha \hat{\theta}+\sin \alpha \hat{\varphi}) e^{j \bar{k}_{i} \cdot \bar{r}}
$$

where $\alpha$ is the polarization angle, $k_{i}$ is the propagation vector given by:

$k_{i}=k_{0}\left(\sin \theta_{i} \cos \varphi_{i} \hat{x}+\sin \theta_{i} \sin \varphi_{i} \hat{y}+\cos \theta_{i} \hat{z}\right)$

and $\hat{\theta}$ and $\hat{\phi}$ are the unit vectors in the spherical coordinate system and $\left(\theta_{i}, \phi_{i}\right)$ defines the angle of arrival of the plane wave. In the three dimensional case, the RCS is defined as [15]:

$R C S(\theta, \varphi)=\lim _{r \rightarrow \infty} 4 \pi r^{2} \frac{\left|E_{s}^{\theta}(r, \theta, \varphi)\right|^{2}+\left|E_{s}^{\varphi}(r, \theta, \varphi)\right|^{2}}{\left|E_{i}\right|^{2}}$

To validate our FDTD program, RCS of a plasma monopole antenna with $f_{p}=70 \mathrm{GHz}$ and collision frequency of $10 \mathrm{GHz}$ is compared with those of CGFFT numerical results which were simulated in [7], as shown in Figure 2. In this comparison we considered the radius of the plasma column $r=5$ $\mathrm{mm}$, dielectric constant of plasma tube $\varepsilon_{r}=3.4$ and thickness of the column as $2 \mathrm{~mm}$. Comparison between our FDTD simulation and CGFFT result shows close agreement between the two different codes.

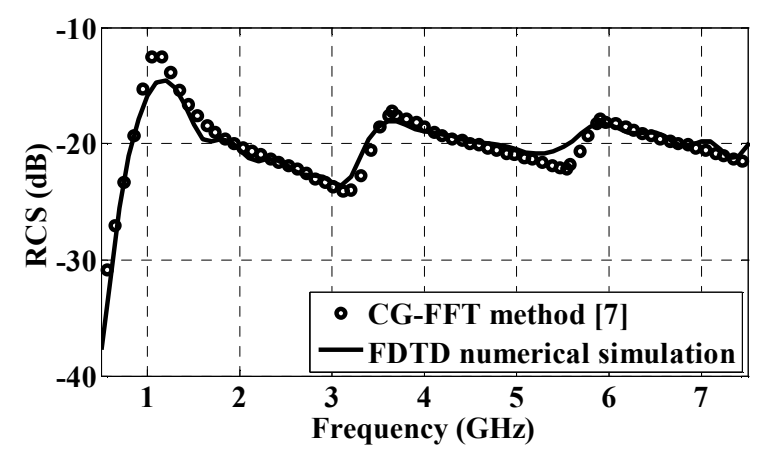

Fig. 2. Comparison between RCS of a plasma column antenna with FDTD numerical simulation and CG-FFT method results.

\section{NUMERICAL RESULTS AND DISCUSSION}

\section{A. The effects of the radius of the plasma column}

Numerical simulation of a plasma antenna, as shown in Figure 3, reveals that in a SWD plasma monopole antenna, similar to a conventional metallic radiator, one method to widen the acceptable operational bandwidth will be to decrease the $L / r$ ratio [16], where $L$ is the length of the plasma antenna and $r$ is the radius of the tube. It has been observed that for a given length of a plasma column, its impedance variations becomes less sensitive as a function of frequency as $L / r$ decreases. Thus, more broadband characteristics can be obtained by increasing the diameter of the plasma tube, similar to metallic conventional antennas. To demonstrate this, in Figure $3 \mathrm{a}$, we have plotted, as a function of frequency, the input impedance of plasma monopole antenna with $L / r=70$, 34 and 23. When the plasma frequency is large enough with respect to the excitation frequency, the imaginary part of the input impedance of plasma antenna can be eliminated by making the total length $L$ of the antenna less than $\lambda / 4$, where $\lambda$ is the wavelength. This value is approximately $0.18 \lambda F^{\prime}$, when the plasma frequency is $7500 \mathrm{MHz}$ and $F^{\prime}=\frac{L / r}{1+L / r}$. However, its value increases by increasing the plasma frequency and consequently conductivity of the plasma while for the maximum conductivity this value becomes $0.24 \lambda F^{\prime}$ [16]. In Figure $3 a$ it is observed that increasing the radius of the column decreases the resistance at the first resonance. The radiation pattern is essentially unaffected by the thickness of the plasma tube in the regions of intense radiation. However, as the radius of the tube increases, the minor lobes diminish in intensity and the nulls are replaced by low level radiation as shown in Figure $3 b$.

\section{B. The effects of the collision frequency}

The collision frequency is a function of pressure and represents the loss mechanism of plasma. It depends on the type of gas and the average number of the particles. There are 
various kinds of collisions in plasma whereas their evaluation is complicated and is not reported here. Readers interested in this subject can refer to [17]. The complex plasma conductivity is stated as:

$$
\sigma(\omega)=\frac{\varepsilon_{0} \omega_{p}^{2}}{j \omega+v}
$$

Where, $\omega_{p}=2 \pi f_{p}=\sqrt{N e^{2} / m \varepsilon_{0}}$ in which $f_{p}$ is the plasma resonant frequency $(\mathrm{Hz}), N$ is the electron density $\left(\mathrm{m}^{-3}\right), v$ is the average collision frequency $(\mathrm{Hz}), \varepsilon_{0}$ is the free space permittivity $(\mathrm{F} / \mathrm{m}), e$ and $m$ are the electron charge (c) and mass $(\mathrm{Kg})$, respectively.

(a)

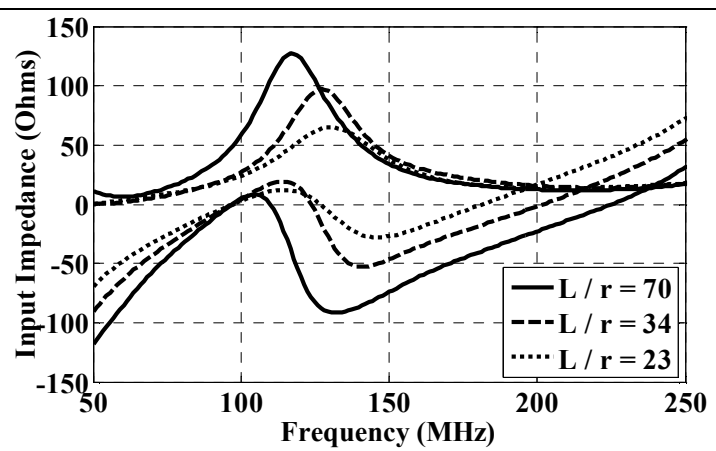

(b)

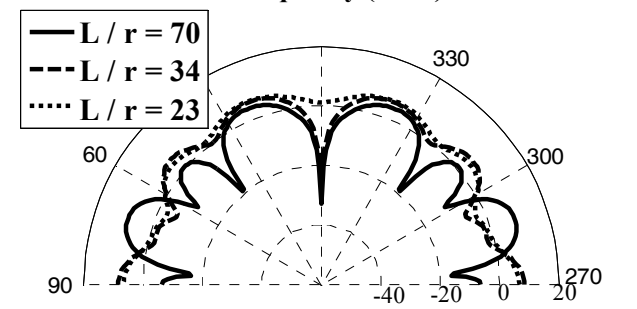

Fig. 3. The effects of the variations of the diameter of plasma tube, in the plasma frequency of $7500 \mathrm{MHz}$ and collision frequency of $400 \mathrm{MHz}$, on (a) input impedance, (b) radiation pattern when the length of antenna is $3 \lambda / 2$.

In a certain plasma frequency the conductivity of the plasma column decreases as the collision frequency increases and it affects all characteristics of a plasma antenna. Increasing the collision frequency from $200 \mathrm{MHz}$ to $1000 \mathrm{MHz}$, in a fixed plasma frequency, decreases the gain of the plasma antenna, as illustrated in Figure 4a, which is due to the increased loss in the plasma column. The gain of a plasma monopole antenna, in our case, is less than that of a conventional metallic antenna and their difference increases as frequency increases. This means that at lower lengths plasma antenna operates more effectively than at higher lengths.

Stealth application of a plasma antenna has high priority over its different applications. Numerical investigation of the impact of collision frequency on the RCS of a plasma antenna, as shown in figure 4.b, reveals that RCS reduces with increasing the collision frequency which is due to the reduced conductivity of the antenna. Therefore, a larger collision frequency is preferable for the purpose of RCS reduction. It is conceivable to optimize the plasma RCS by controlling the plasma collision frequency for practical applications. In our simulations, the incident plane wave is with $\theta_{i}=180^{\circ}, \phi_{i}=0^{\circ}, \alpha=0^{\circ}$.

(a)
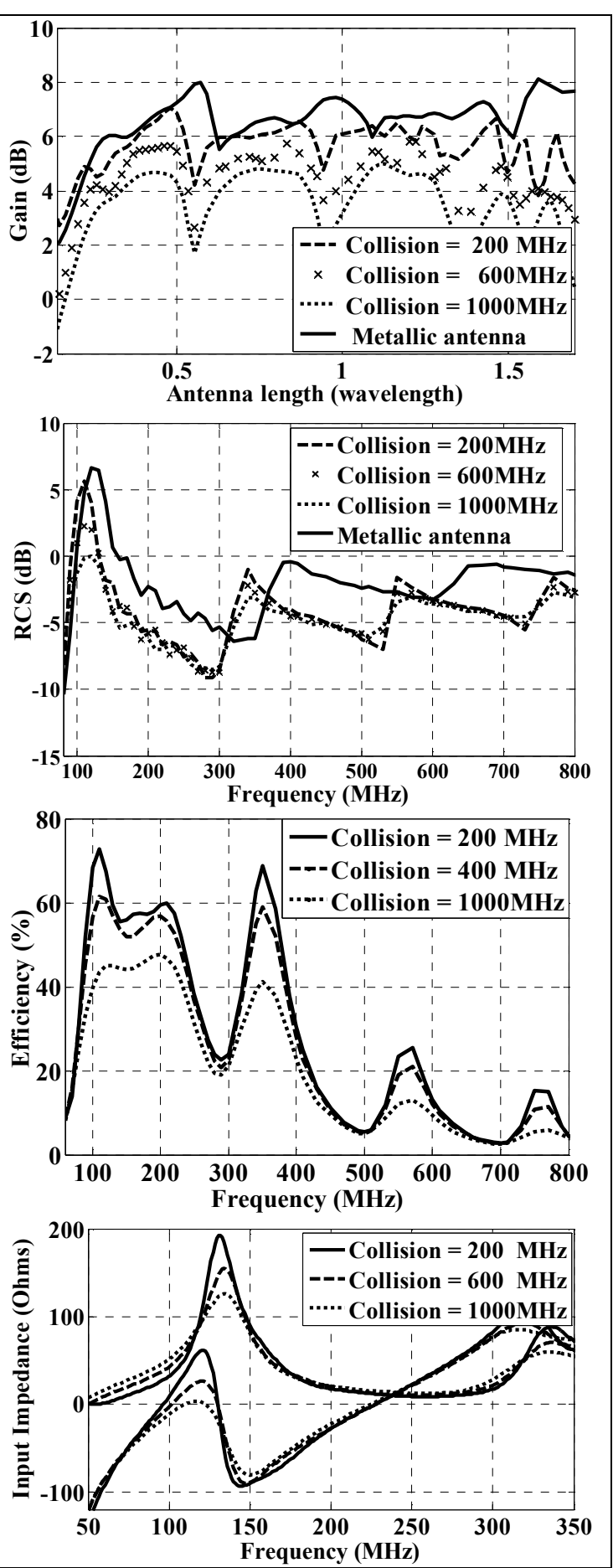

Fig. 4. Characteristics of a plasma antenna when the plasma frequency is $f_{p}=7500 \mathrm{GHz}$ and at different collision frequencies (a) gain, (b) RCS, (c) Efficiency and (d) Input impedance. 
The efficiency of the plasma antennas is a key parameter to evaluate the possibility of using a plasma element instead of a metallic one. The efficiency takes into account the losses at the input terminal and the structure of the antenna and is directly related to plasma conductivity. Due to the much lower conductivity of the plasma, at higher collision frequencies the efficiency of the antenna decreases, as shown in Figure 4c.

It is noted that the radiation pattern of a plasma antenna is essentially unaffected by the variation of the collision frequency. However, as the collision frequency increases, the minor lobes diminish in intensity and the nulls are replaced by low level radiation. When the plasma frequency is small, at low collision frequencies, the input impedance of the antenna has a lot of oscillations which decrease when collision frequency increases. The collision loss of plasma increases the loss of the antenna, resulting in a reduced radiated power and damping the impedance variations. Therefore, increasing the collision frequency increases the bandwidth of the plasma antenna. However, the collision frequency does not influence the period of resonance as shown in Figure 4d. This may be considered as one of the useful methods which can be used for a dynamic control of an antenna bandwidth by varying the Q-factor of the antenna.

\section{The effects of the plasma frequency}

Measurements of plasma electron number density using Langmuir probes have proved that the increase of input RF power at a fixed filling gas pressure increases the plasma density in the column $[18,19]$ and plasma frequency is a function of plasma density. Increasing the plasma frequency in the column increases the conductivity and consequently it causes considerable increment in the values of the gain, RCS and efficiency of a plasma antenna. At higher plasma frequencies with respect to the excitation frequency, the gain and the RCS of a plasma antenna are as high as of a metallic antenna. The variations of the gain, RCS and efficiency of a plasma antenna versus the plasma frequency, in the collision frequency of $400 \mathrm{MHz}$, are shown in Figures 5a-5c.

Comparison between the radiation patterns of a plasma antenna with $L=3 \lambda / 2$ at different plasma frequencies with a metallic antenna (Figure 5d) shows that in the plasma antenna, the numbers of lobes are increased up to those of a conventional metallic antenna when the plasma frequency increases and also that the radiation pattern finds deeper nulls. At higher plasma frequencies, the radiation pattern becomes more similar to that of a metallic one. Furthermore, an interesting phenomenon is observed when $f_{p} / f<2.5$. Whereas the metallic monopole antenna does not radiate energy along the monopole axis, the plasma antenna radiates thus showing the characteristics of an end fire antenna, as was introduced before in [4] and [20]. This occurs because the radiating source is a surface wave along the column. The plasma column does not carry a current, but a surface wave propagates along the column and reaches its tip. Therefore, it contributes the radiation towards the end fire direction, as shown in Figure 5d and increasing the collision frequency, increases this property. This narrow cone beam is associated with the least antenna efficiency as was shown in Figure 5c (the dotted line where $f_{p}=1500 \mathrm{MHz}$ ), so it is not of practical interest. Numerical simulation reveals that the input impedance curves of the antenna, as shown in Figure 5e, are altered as the plasma frequency varies. This property can be used to construct dynamically reconfigurable antennas.

\section{A linear array of two plasma elements}

Essential background for this section is covered in the previous sections and the 'elements' are SWD plasma monopole antennas with a radius of $12 \mathrm{~mm}$ and a length of $600 \mathrm{~mm}$ at the plasma frequency of $7500 \mathrm{MHz}$ and the collision frequency of $400 \mathrm{MHz}$. A broadside array of two parallel side-by-side, equal in phase current elements has been analyzed numerically. Let $d$ be the separation of elements. Referring to the arrangement of Figure $6 \mathrm{a}$, the mutual resistance $R_{12}$ and reactance $X_{12}$ of the first resonant frequency of the antenna are presented in Figure $6 \mathrm{~b}$ as a function of the spacing $d$. Moreover, the quantity $R_{11}+R_{12}$, which is important in this array calculation is also illustrated, where $R_{11}$ is the self impedance of the elements. It is worthy to mention that at the spacing $0.45<d<0.52$ between the elements, the total resistance is in its minimum value around 28.5; which is incorporated with the maximum array gain as shown in Figure 6c.

\section{CONCLUSION}

Numerical simulation of the characteristics of a plasma column antenna, when the plasma density is uniform in the column, has been established in this paper. Numerical simulation was validated through comparisons with other investigations. It has been shown that for a fixed excitation power, the variation of gas pressure, which varies the collision frequency, changes antenna characteristics. Increasing the collision frequency decreases antenna gain, RCS, efficiency and peak input impedance and it may be considered as one of the useful methods which can be used for a dynamic control of an antenna bandwidth. From the computed numerical results it is clear that increasing the excitation power, which increases the plasma frequency, increases the conductivity and consequently it causes considerable increment in the values of gain, RCS and efficiency of the antenna. Moreover, it can be considered as a powerful tool to construct dynamically reconfigurable antennas because the impedance curves are altered as plasma frequency varies. Variations of the radii of the plasma column have non negligible effects on the antenna characteristics. Increasing the radius of the tube can be considered as a method to widen the acceptable operational bandwidth of the antenna and replacing the nulls in the radiation pattern by low level radiation. Finally, we have presented the mutual impedance and gain of a broadside array of two parallel side-by-side, equal in phase current SWD plasma monopole antenna and it was concluded that maximum gain will be achieved when the spacing between elements is around $\lambda / 2$. 


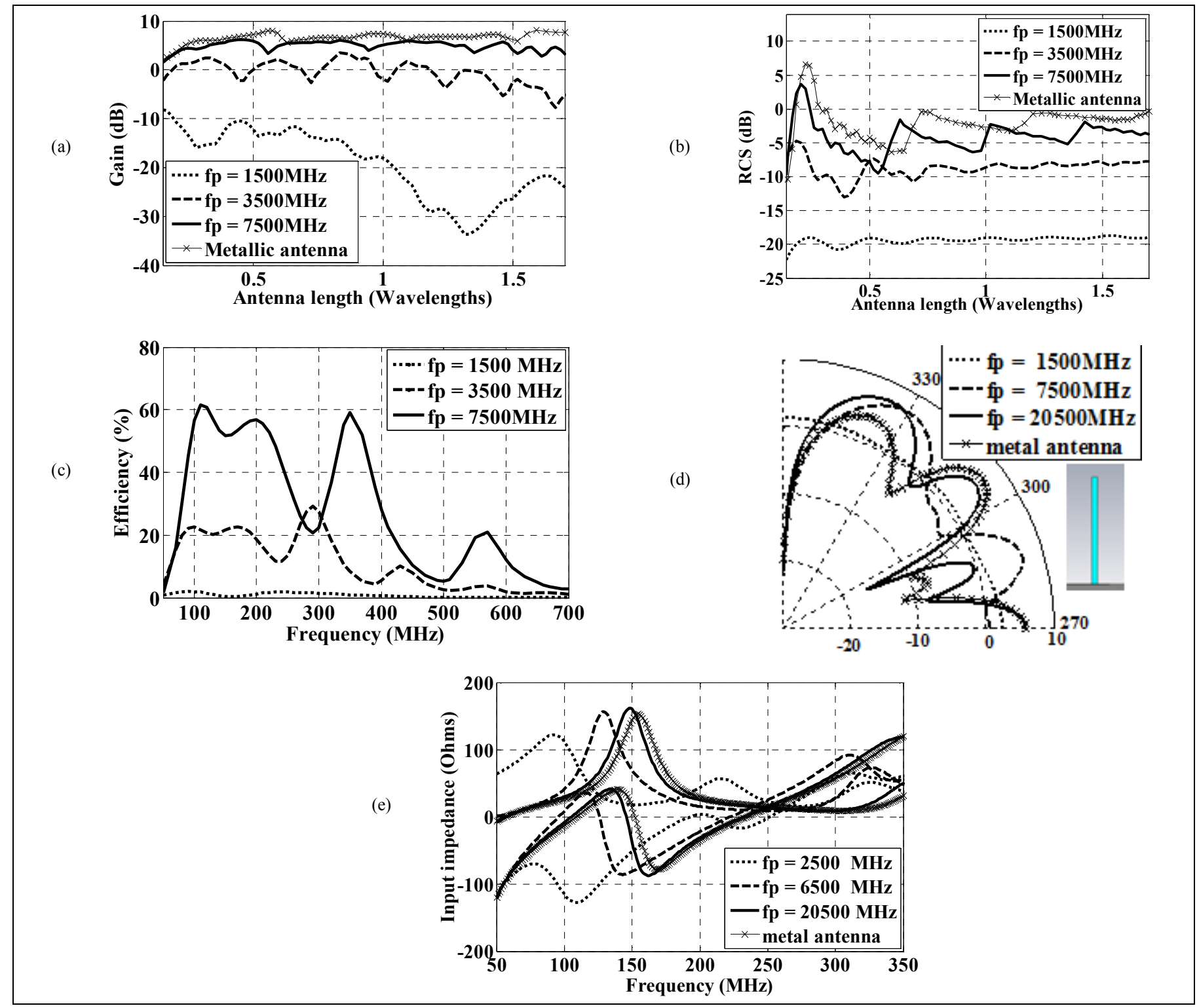

Fig. 5. The effects of the plasma frequency on the characteristics of a plasma antenna at the collision frequency of $400 \mathrm{MHz}$ ( (a) gain, (b) RCS, (c) efficiency, (d) radiation pattern and (e) input impedance.

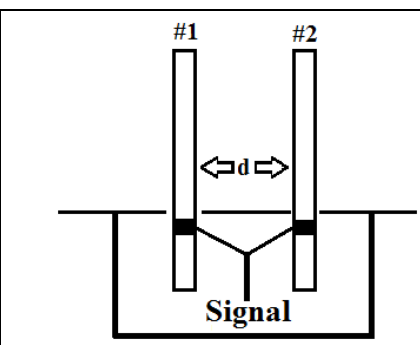

(a)

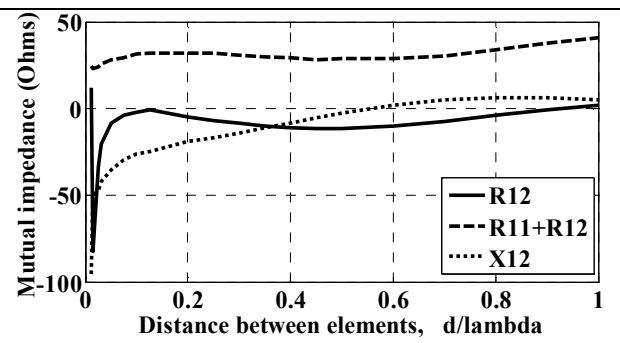

(b)

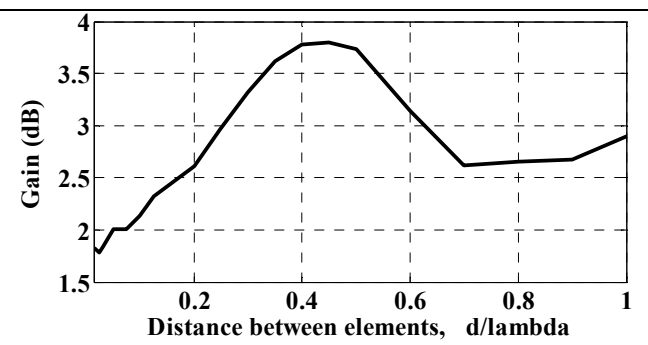

(c)

Fig. 6. (a) Arrangement of an array of two parallel side-by-side SWD plasma elements, (b) mutual impedance of the broadside array as a function of separation between elements and (c) gain of the array. 


\section{REFERENCES}

[1] J. P. Rayner, A. P. Wichello, A. D. Cheetham, "Physical characteristics of plasma antennas", IEEE Transactions on Plasma Science, Vol. 32, No. 1, pp. 269-281, 2004

[2] G. Cerri, R. De Leo, V. M. Primiani, P. Russo, "Measurement of the properties of a plasma column used as a radiating element", IEEE Transactions on Instrumentation and Measurement, Vol. 57, No. 2, pp. 242-247, 2008

[3] T. Anderson, I. Alexeff, E. Farshi, N. Karnam, E. P. Pradeep, N. R. Pulasani, J. Peck, “An operating intelligent plasma antenna”, 16th IEEE International Pulsed Power Conference, Albuquerque, New Mexico USA, pp. 353-356, 2007

[4] Y. Lee, S. Ganguly, "Analysis of a plasma-column antenna using FDTD method", Microwave and Optical Technology Letters, Vol. 46, No. 3 , pp. 252-259, 2005

[5] Z. H. Qian, R. S. Chen, K. W. Leung, H. W. Yang, "FDTD analysis of microstrip patch antenna covered by plasma sheath", Progress in Electromagnetics Research, Vol. 52, pp. 173-183, 2005

[6] P. Russo, G. Cerri, E. Vecchioni, "Self consistent analysis of cylindrical plasma antennas", IEEE Transactions on Antenna and Propagation, Vol. 59, No. 5, pp. 1503-1511, 2011

[7] Z. H. Qian, R. S. Chen, Z. H. Fan, P. L. Rui, "Analysis of electromagnetic scattering from plasma antenna using CG-FFT method", International Journal of Infrared and Millimeter Waves, Vol. 29, No. 5, pp. 486-492, 2008

[8] F. Sadeghikia, F. Hodjat-Kashani, J. Rashed-Mohassel, J. GhayoomehBozorgi, "Characterization of a surface wave driven plasma monopole antenna", Journal of Electromagnetic Waves and Applications, Vol. 26, No. 2-3, pp. 239-250, 2012

[9] Oleg A. Popov, High density plasma sources: design, physics and performance, Noyes publication, Park Ridge, New Jersey, 1995

[10] R. Kumar, D. Bora, "Experimental study of parameters of a plasma antenna", Plasma Science and Technology, Vol. 12, No. 5, pp. 592-600, 2010
[11] Z. Rakem, P. Leprince, J. Marec, "Characteristics of a surface wave produced discharge operating under standing wave conditions", Revue Physique Appliquee, Vol. 25, No. 1, pp. 125-130, 1990

[12] L. J. Nickisch, P. M. Franke, "Finite-difference time-domain solution of Maxwell's equations for the dispersive ionosphere", IEEE Antennas and Propagation Magazine, Vol. 34, No. 5, pp. 33-39, 1992

[13] K. S. Kunz, R. J. Rubbers, The finite difference time domain method for electromagnetics, CRC press, Boca Raton, FL, 1993

[14] A. Taflove, Computational electromagnetics: The finite difference time domain method, Artech House, Boston, FL, 1995

[15] R. J. Luebbers, K. S. Kunz, M. Schneider, F. Hansberger, “A finitedifference time-domain near zone to far zone transformation [electromagnetic scattering]", IEEE Transactions on Antennas and Propagation, Vol. 39, No. 4, pp. 429-433, 1991

[16] A. Balanis, Antenna theory, analysis and design, John Wiley \& Sons Inc., New Jersey, 2005

[17] U. Kortshagen, C. Busch, L. D. Tsendin, “On simplifying approaches to the solution of the Boltzmann equation in spatially inhomogeneous plasmas", Plasma Sources Science and Technology, Vol. 5, No. 1, pp. 117, 1996

[18] M. Y. Naz, A. Ghaffar, N. U. Rehman, S. Naseer, M. Zakaullah, "Double and triple Langmuir probes measurements in inductively coupled nitrogen plasma”, Progress In Electromagnetics Research, Vol. 114, pp. 113-128, 2011

[19] M. Y. Naz, A. Ghaffar, N. U. Rehman, M. Azam, S. Shukrullah, A. Qayyum, M. Zakaullah, "Symmetric and asymmetric double Langmuir probes characterization of radio frequency inductively coupled Nitrogen plasma”, Progress in Electromagnetics Research, Vol. 115, pp. 207-221, 2011

[20] I. L. Morrow, J. R. James, "Fundamental limitation on excitation of a surface wave on a plasma column", IEEE Antennas and Propagation Society International Symposium, Texas, USA, Vol. 4, pp. 272-275, 2002 